Jürgen Aring und Manfred Sinz

\title{
Neue Leitbilder der Raumentwicklung
}

\author{
Ein Impuls zur Modernisierung der Raumordnung?
}

\section{New concepts for spatial development}

An impulse for the modernisation of spatial planning?

\section{Kurzfassung}

Mit den drei Schwerpunkten „Wachstum und Innovation“, „Daseinsvorsorge sichern“ und „Ressourcen bewahren, Kulturlandschaften gestalten“ wurden 2006 nach einem mehrjährigen fachlichen und politischen Diskussionsprozess neue Leitbilder der Raumentwicklung für Deutschland beschlossen. Der Beitrag stellt dar, warum die neuen Leitbilder als Impuls zur Modernisierung der Raumordnung in Deutschland zu verstehen sind. Dabei stehen zwei Thesen im Vordergrund: Erstens identifizieren die neuen Leitbilder Handlungsschwerpunkte nicht aus einer Binnenperspektive der Raumordnungstradition, sondern aus dem aktuellen gesellschaftlichen Umfeld. Zweitens bestimmen politische Aufgaben statt Raumordnungskategorien das Denken. Damit wird implizit von einer fast automatisierten, aber sachlich nicht mehr angemessenen Ausrichtung der Raumentwicklungspolitik vorrangig zugunsten ländlicher Räume abgerückt und der Entwicklungsperspektive von Stadtregionen eine gleichberechtigte Bedeutung zugemessen. Der Beitrag reflektiert den davon ausgelösten und noch nicht abgeschlossenen wissenschaftlichen und politischen Diskurs.

\begin{abstract}
After an intensive discussion within the planning community as well as in the political area the Standing Conference of Ministers responsible for Spatial Planning has decided upon new visions and guidelines for spatial development in Germany. Its main topics are contributions of planning and regional policy to general social and economic tasks like "growth and innovation", "guaranteeing public services" and "safeguarding natural resources" as well as "shaping cultural landscapes". The paper wants to explain why the new guidelines indicate an innovative step for spatial planning at the federal level in Germany. The main messages are: The guidelines identify their goals and proposals for action not from a traditional territorial perspective using categories like agglomerations and rural areas. Instead the new strategies refer to prevailing economic, social and environmental challenges like regional competitiveness, demographic change and conflicts on land-use priorities. This signifies a paradigm shift from traditional equity-oriented interventions in favour of rural areas to a stronger emphasis on urban agglomerations and their metropolitan development potentials. The apparent or supposed shift led to continuing political and ideological discussions which are treated in this article.
\end{abstract}




\section{Einführung}

Ende Juni 2006 hat die Ministerkonferenz für Raumordnung (MKRO) nach einem mehrjährigen fachlichen und politischen Diskussionsprozess neue Leitbilder der Raumentwicklung für Deutschland beschlossen. Mit den Überschriften „Wachstum und Innovation“, "Daseinsvorsorge sichern" und „Ressourcen bewahren, Kulturlandschaften gestalten " werden die angestrebten Schwerpunkte raumordnerischen Handelns für die nächsten Jahre benannt.

Die sich abzeichnenden Leitbilder - und zwar die Texte genauso wie die Leitbildkarten - haben bereits in ihrer Entstehungsphase fachliches und politisches Interesse geweckt. Die Organisatoren des Leitbilddiskurses mussten dabei nicht eigens um Aufmerksamkeit werben, sondern konnten auf Diskussionsbeiträge, Anfragen und Kommentare reagieren. Dabei kamen die Reaktionen nicht nur aus der engeren Fachgemeinschaft der Raumordner, sondern auch von außerhalb. So meldeten sich sowohl Interessenvertreter ländlicher Räume als auch Befürworter bzw. Kritiker des Metropolregionenkonzepts zu Wort. Teilweise waren die Kommentare sehr grundsätzlich, etwa in der Frage der Gleichwertigkeit der Lebensverhältnisse.

Angesichts des Aufmerksamkeitsdefizits, über das die Raumordnung immer wieder klagt, kann man über die erfolgten Rückkopplungen im Leitbildprozess durchaus zufrieden sein. Wichtiger ist jedoch zu fragen, warum es zu den intensiven Diskussionen und Reaktionen kam. Wir vermuten, dass die Thesen im Leitbilddiskurs und die Inhalte der neuen Leitbilder einerseits gewohnte Positionen in Frage stellen und andererseits ein neues Interesse an räumlichen Entwicklungsstrategien geweckt haben. Im Leitbildprozess wurde eine Modernisierung der Raumordnung eingefordert, und mit dem Ergebnis wurden substanziell neue Sichtweisen und Arbeitsschwerpunkte beschlossen. Solche Veränderungen wecken auch Widerstände und polarisieren gegebenenfalls. So fühlten sich viele durch den Leitbildprozess und seine Ergebnisse herausgefordert, Position zu beziehen - teils zustimmend, teils ablehnend, aber in jedem Fall engagiert.

Die drei neuen Leitbilder der Raumentwicklung sind - so unsere These - in mehrfacher Hinsicht innovativ für die Raumordnung. Sie identifizieren Handlungsschwerpunkte aus dem gesellschaftlichen Kontext und nicht aus einer Binnenperspektive der Raumordnungstradition. Sie setzen neue Akzente zwischen und in den Handlungsfeldern Entwicklung, Ausgleich und Ordnung. Sie stellen Aufgaben in den Vordergrund und nicht Raumkategorien. Und schließlich richten sich die neuen Leitbilder nicht nur an Raumordnung und Lan- desplanung, sondern suchen Synergien mit Fachpolitiken, mit der Wirtschaft und der Zivilgesellschaft. Im Hinblick auf die Handlungsorientierung betonen die Leitbilder gemeinsame Interessen unterschiedlicher Akteure.

Für die Raumordnung sind Diskussionen über solche Schwerpunktverschiebungen nicht neu. Schon in den 1970er Jahren löste ein Gutachten der Kommission für wirtschaftlichen und sozialen Wandel (1977) eine heftige politische Diskussion darüber aus, ob erkennbare Tendenzen $\mathrm{zu}$ einer großräumig funktionalen Arbeitsteilung unterstützt oder bekämpft werden sollten. Abgesehen von der damals weitgehend ungeklärt gebliebenen Maßstabsfrage (innerhalb welcher Raumeinheiten mit welcher Ausdehnung sollte Arbeitsteilung „erlaubt" sein?), drehte sich die Auseinandersetzung im Kern darum, ob Interventionen bzw. steuernde Eingriffe des Staates zugunsten benachteiligter Regionen aus Gründen der Solidarität und des sozialen Ausgleichs angezeigt oder ökonomisch kontraproduktiv seien. Im Ergebnis des Gutachtens blieben diese Frage strittig und der Diskurs ohne Folgen für die Programme und Pläne der Raumordnung. Im Gegensatz dazu ist aus dem Leitbildprozess mit seiner ebenfalls grundsätzlichen Diskussion ein politischer Beschluss hervorgegangen.

Die Akzentsetzungen aus dem Leitbildprozess muss man vor der seit langem schwelenden „zweiflerischen und nagenden Diskussion über den Stellenwert unseres Faches" (Sinz 2002, S. 57) sehen, wie sie exemplarisch beim von ARL und BBR ausgerichteten Zukunftsforum RaumPlanung im Jahr 2001 spürbar war. Manfred Sinz äußerte seinerzeit folgende These: Die Raumordnung „schwankt zwischen Extremen und neigt zu Polarisierungen. Raumordnung sieht sich entweder als allzuständige Koordinationsdisziplin oder als ohnmächtige Mahninstanz. Sie schwankt bei vermeintlich oder tatsächlich neuen Entwicklungen zwischen Hysterie (häufiger) und Ignoranz (seltener). Man gefällt sich bis in die politischen Spitzen in zynischem Defätismus oder pflegt auf Arbeitsebene eine traurige Mutlosigkeit. Die Zukunft erscheint entweder unausweichlich oder völlig unvorhersehbar. Konzepte setzen entweder auf Ordnung oder Entwicklung, entweder auf Wettbewerb und Markt oder Intervention und Ausgleich. Bei den Akteuren der Raumplanung stehen hemdsärmelige Macher zaudernden Bedenkenträgern verständnislos gegenüber. Einige versprengte Optimisten verachten den Pessimismus der vielen Apokalyptiker. Visionäre und Prediger ignorieren die Fleißarbeit der Analytiker. Die nüchternen, aufgeklärten Skeptiker sind in der Minderzahl." (ebda.) 
Wenn dieser Befund einigermaßen stimmt, dann muss man fragen, wie es zu einem Beschluss über neue Leitbilder der Raumentwicklung kommen konnte, der über eine „Buchhaltung von Fachplanungen“ (Ganser 1983, S. 62) und eine Betonung der „bewährten Ansätze der Raumplanung", wie sie häufig in Beiratsempfehlungen und Arbeitskreispapieren von Akademien und Fachgremien anzutreffen ist, deutlich hinausgehen konnte. Zwei Antworten scheinen denkbar: erstens: „Die Zeit war reif" und zweitens: „Die neuen Leitbilder sind in einem Diskurs entstanden“.

Vor diesem Hintergrund stellt der folgende Beitrag einige angestrebte Innovationen für die Raumordnung vor, die ihren Niederschlag in den neuen Leitbildern gefunden haben. Es wird zu zeigen versucht, was neu ist und wie es diskutiert wurde. Zum besseren Verständnis wird vorab in knapper Form der Leitbildprozess dargestellt. Für eine zusammenfassende Darstellung der Inhalte der Leitbilder wird auf den Beitrag von Horst Lutter in diesem Heft verwiesen.

Zur Einordnung des vorliegenden Beitrags ist folgende methodologische Bemerkung einzuschieben: Die Verfasser waren intensiv in die Entwicklung der neuen Leitbilder eingebunden. So sind die nachfolgenden Überlegungen zwar reflexiv angelegt, aber dennoch aus einer Binnenperspektive geschrieben. Der Beitrag ist deshalb nicht nur ein Dokument über wichtige Resultate des Leitbildprozesses, sondern auch aus dem Leitbildprozess. Es bleibt unbeteiligten Autoren vorbehalten, den Prozess und seinen Innovationscharakter aus einer neutraleren Position aufzuarbeiten.

\section{Entstehung und Zielrichtung der neuen Leitbilder}

Seit der Neufassung des Raumordnungsgesetzes 1997 sind Leitbilder explizit als Instrument zur Abstimmung der räumlichen Entwicklungsvorstellungen von Bund und Ländern vorgesehen. Die Aufnahme eines Auftrags zur Erarbeitung von Leitbildern in das Raumordnungsgesetz greift die positiven Erfahrungen auf, die zu Beginn der 1990er Jahre mit dem Raumordnungspolitischen Orientierungsrahmen (BMBau 1993) gemacht wurden. Er wurde seinerzeit verstanden als „ein Neuansatz, ein neuer Weg, um raumordnungspolitische Rahmenaussagen zu treffen, in Abkehr von dem System eines Bundesraumordnungsprogramms“ (Schwaetzer 1994, S. 6). Der Raumordnungspolitische Orientierungsrahmen formulierte räumliche Leitbilder als Strategiekonsens zwischen Bund und Ländern angesichts der Herausforderungen der Deutschen Einheit und trug der Einsicht Rechnung, dass „eine Raumord- nungskonzeption für das gesamte Bundesgebiet nicht aus der der bloßen Summierung von 16 Landesentwicklungsplanungen gebildet werden kann" (ebenda, S. 5). Er wurde später durch einen Raumordnungspolitischen Handlungsrahmen (BMBau 1995) ergänzt, dessen Aufgabenstellungen wie z.B. die Bildung von Städtenetzen in den Jahren danach im Rahmen von Modellvorhaben der Raumordnung angegangen wurden.

$\mathrm{Zu}$ Beginn des neuen Jahrzehnts waren die im Raumordnungspolitischen Orientierungsrahmen und im entsprechenden Handlungsrahmen angelegten Aufgaben einerseits weitgehend abgearbeitet, andererseits hatten sich die Rahmenbedingungen der Raumentwicklung seit Beginn der 1990er Jahre deutlich verändert bzw. waren neu zu deuten. Vor diesem Hintergrund entschloss sich das zuständige Bundesministerium (seinerzeit Bundesministerium für Raumordnung, Bauwesen und Städtebau), die Grundlagen für ein Nachfolgedokument erarbeiten zu lassen. Anders als beim Raumordnungspolitischen Orientierungsrahmen, der 1992 in einem verwaltungsinternen Prozess zwischen Bund und Ländern entstand, sollten die neuen Leitbilder in einem fachöffentlichen Diskurs entwickelt werden. Rückblickend kann man von einem zweistufigen, sich teilweise überlappenden Prozess mit vielen Rückkopplungen sprechen:

(1) Ein stärker fachlich-wissenschaftlich geprägter Diskussionsprozess entstand im Rahmen eines auf knapp zwei Jahre angelegten Begleitprojekts im Rahmen der Ressortforschung, das nach öffentlicher Ausschreibung an einen Berater vergeben wurde. ${ }^{1}$ Im Rahmen dieses Projekts (vgl. die ausführlichen Darstellungen in Aring/ Sinz 2006 und Aring 2006) wurde ein fachlich-wissenschaftlicher Prozess organisiert, dessen zentrale Elemente mehrere Workshops, das Aufgreifen der Fachdiskussion, die Auseinandersetzung mit internationalen Impulsen, die Vernetzung mit der Erarbeitung des Raumordnungsberichts, die Verknüpfung von Diskursund Kreativphasen und schließlich die Produktion von Texten und Karten waren. So entstand in enger Zusammenarbeit zwischen dem Bundesministerium, dem Bundesamt für Bauwesen und Raumordnung (BBR) und dem Berater ein Grundlagendokument, das im September 2005 auf einer Fachtagung in Bonn diskutiert wurde und im Folgenden zur Plattform einer weitergehenden Diskussion und Abstimmung im Rahmen der Ministerkonferenz für Raumordnung (MKRO) wurde.

(2) Parallel dazu gab es einen stärker fachlich-politisch geprägten Diskussionsprozess, dessen Eckpunkte die Vorgabe der drei Leitbildthemen durch die MKRO im April 2005 und der endgültige Beschluss der neuen Leit- 
bilder im Juni 2006 bilden. Elemente des politischen Diskurses waren die Stellungnahme der Bundesregierung zum Raumordnungsbericht, die Empfehlungen des Beirats für Raumordnung, die teilweise Abstimmung mit Fachpolitiken und Interessenträgern auf Bundesebene sowie insbesondere die Abstimmung zwischen Bund und Ländern in den Gremien der MKRO.

Im Sommer 2006 wurden die neuen Leitbilder von der Ministerkonferenz für Raumordnung verabschiedet und vom Bundesministerium für Verkehr, Bau und Stadtentwicklung publiziert. In den Vorbemerkungen heißt es: „Als Brücke zwischen den raumbezogenen politischen Zielsetzungen, den verbindlichen Festlegungen der Raumordnungspläne und konkreten Projekten der Handlungsebene beschreiben die drei Leitbilder

- ,Wachstum und Innovation'

- ,Daseinsvorsorge sichern' und

- ,Ressourcen bewahren, Kulturlandschaften gestalten'

die Aufgabenschwerpunkte der Raumordnung in den nächsten Jahren“ (BMVBS 2006, S. 1).

Die Ministerkonferenz für Raumordnung will mit den Leitbildern auch einen Beitrag Deutschlands zur Diskussion über eine gemeinsame europäische Raumentwicklungspolitik leisten. Im Leitbildbeschluss vom 30. Juni 2006 (MKRO 2006) heißt es: Sie (die Ministerkonferenz, die Verf.) misst der Ergänzung wirtschaftlicher und sozialer Komponenten der europäischen Kohäsionspolitik um die territoriale Dimension durch ausgewogene Verknüpfung dieser drei Belange untereinander eine hohe Bedeutung bei. Sie erwartet, dass die Leitbilder und Handlungsstrategien Impulse geben, die

- alle Räume befähigen, ihre Stärken zu stärken, ihre Kräfte und Potenziale zu bündeln und zu vernetzen sowie die gemeinsame partnerschaftliche Verantwortung von Regionen zu entwickeln;

- die tragende Rolle der europäischen Metropolregionen in Deutschland stärken sowie die Weiterentwicklung von Kooperationsformen zu großräumigen Verantwortungsgemeinschaften unterstützen;

- die Neuausrichtung von Strategien, Standards und Instrumenten der Raumordnung unterstützen, um auch künftig wertgleiche Lebensverhältnisse zu gewährleisten, was insbesondere für die Bereitstellung und Erreichbarkeit von Dienstleistungen und Infrastrukturen in allen Teilräumen Deutschlands gilt, und
- die verstärkte Koordinierung der raumwirksamen Fachpolitiken mit den Erfordernissen der Raumordnung befördern.“

\section{Innovation I: Die Kontexte neu deuten - Offenheit für die Außensicht}

Im Leitbildprozess wurde von den Organisatoren des Diskurses die These vertreten, Raumentwicklungspolitik habe - im Rahmen des im Raumordnungsgesetz formulierten Nachhaltigkeitsziels - eine den gesellschaftlichen Entwicklungserfordernissen dienende Funktion zu erfüllen. Die Ziele und Grundsätze der Raumordnung von Bund und Ländern seien deshalb nicht absolut und statisch, sondern bedürften unter veränderten gesellschaftlichen Rahmenbedingungen einer Überprüfung und ggf. Neuausrichtung insbesondere von Prioritäten. Dieses moderne Rollenverständnis der Raumordnung und Raumentwicklung war schon im Raumordnungspolitischen Orientierungsrahmen 1993 erkennbar. Dort heißt es: „Der Raumordnungspolitische Orientierungsrahmen greift vor dem Hintergrund der dramatisch veränderten räumlichen Rahmenbedingungen die im Raumordnungsgesetz des Bundes verankerten Ziele und Grundsätze dynamisch auf" (BMBau 1993, S. 1).

In vergleichbarem Sinne wurde im Leitbilddiskurs ein Bezug $\mathrm{zu}$ den aktuellen gesellschaftlichen Aufgabenstellungen der Gegenwart hergestellt, nämlich der Schaffung von Arbeitsplätzen in Verbindung mit der Rückkehr zu einer dynamischen wirtschaftlichen Entwicklung einerseits und dem Umgang mit den Anpassungslasten des Umbaus staatlich gesicherter Daseinsvorsorge andererseits. Da diese ökonomisch-gesellschaftlichen Prozesse auch räumliche Seiten haben, sei Raumordnung und Raumentwicklungspolitik gefordert, ihre Aufgabenschwerpunkte und Handlungsansätze entsprechend auszurichten.

In den Diskussionen spitzte sich dieser Zugang auf folgende Frage zu: Soll Raumordnung Entwicklungstrends begleiten, indem für die Gesellschaft nützliche Entwicklungen unterstützt und problematische Entwicklungen abgefedert bzw. aktiv begleitet werden? Oder soll Raumordnung aus einem eigenen Werte- und Aufgabenverständnis heraus agieren, das einen Gegenpol zu den ablaufenden Raumentwicklungsprozessen darstellt und deswegen einen konsequenten Gegenentwurf zu den räumlichen Ausdifferenzierungs- und Polarisierungsprozessen darstellen muss? Die erste Perspektive bemüht sich um eine Neuorientierung bzw. Modernisierung, während die zweite für ein eher 
traditionelles Verständnis von Raumplanung steht. Das klingt zunächst nach sachlichen Handlungsoptionen, doch dahinter verbergen sich nicht nur fachliche Bewertungen, sondern unterschiedliche politische Grundsatzpositionen.

Im Diskurs wurde zweierlei angestrebt. Einerseits wurde die polarisierte Diskussion mit ihren klaren, zum Teil aber auch auf Diffamierung angelegten Begriffen (z. B: „Huldigung an einen neoliberalen Zeitgeist“) positiv aufgegriffen und zugespitzt. Es erschien sinnvoll, offene politische Grundsatzfragen zu benennen und sie einer transparenten Diskussion auszusetzen. Andererseits wurde auch darauf verwiesen, dass die Welt sich letztlich nicht so schwarz-weiß darstellt, wie es die zugespitzte Diskussion erscheinen lässt. Auf die Frage, „ob der Staat nun fürsorglicher Gewährleister oder anstoßender Ermunterer sein soll“ (Sinz 2002, S. 58), kann man letztlich nur antworten: Er muss beides leisten. Zeitgemäße Antworten liegen in einer Kombination der Ansätze, die allerdings Prioritäten für Instrumente und Mitteleinsatz im jeweiligen Zielbereich setzen muss und nicht auf ein ,allen überall alles“ hinauslaufen darf.

Im Leitbildprozess wurde das Prinzip des Sowohl-alsauch durchaus ernst genommen, indem die Leitbilder immer weiter angereichert und ausdifferenziert wurden. Das gilt sowohl für das Leitbild „Wachstum und Innovation“, das im Laufe des Prozesses seine Metropolenlastigkeit verlor, wie für das Leitbild „Sicherung der Daseinsvorsorge“, das neben normativen Konzentrationskonzepten auch die Sicherung von Mindeststandards und flexible Formen der Gewährleistung von Daseinsvorsorge thematisiert. Dabei handelte es sich nach Einschätzung der Verfasser nicht lediglich um Formelkompromisse zur Verknüpfung gegensätzlicher Positionen, sondern um echte inhaltliche Weiterentwicklungen.

\section{Innovation II: Politische Aufgaben statt Raumkategorien im Fokus}

Eine griffige Formulierung aus dem Leitbildprozess lautet: "Stärken stärken“. Sie gibt die Handlungsrichtung im Leitbild "Wachstum und Innovation" an und richtet sich gleichermaßen an Räume mit sehr guten wirtschaftlichen Perspektiven wie an Räume mit Strukturschwächen. Mit dieser Ausrichtung ist das Leitbild "Wachstum und Innovation“ zum Ausgangspunkt einer veränderten raumordnerischen Positionierung geworden, die Aufgaben statt Raumkategorien in den Fokus stellt.
Solange raumordnerisches Denken durch das Erkennen von Disparitäten und das Handeln durch Vorschläge für Maßnahmen zum Disparitätenabbau bestimmt wird, ist jeder Raum gedanklich und analytisch $\mathrm{zu}$ strukturieren: einerseits in zurückhinkende, unterstützungsbedürftige Regionen und andererseits in Räume, die "gebremst" werden müssen oder zumindest nicht gefördert werden dürfen. In der Geschichte der deutschen Raumordnung ist dieses Ziel des Disparitätenabbaus eine enge Verbindung mit Raumkategorien eingegangen. So ist Strukturschwäche zu einem Dauerattribut des ländlichen Raums geworden, ungeachtet dessen tatsächlicher Situation, die sich immer weiter ausdifferenzierte, worauf bereits der Raumordnungsbericht 2000 (BBR 2000) deutlich hinwies.

Historisch gesehen hat dieser besondere Blick auf den ländlichen Raum durchaus seine Berechtigung. StadtLand-Gegensätze waren in der Bundesrepublik der 1950er und 60er Jahre stark ausgeprägt, bevor sie dann im Zuge einer generellen Urbanisierung des Landes zunehmend verblassten (von der Heide 1994, S. 25). In der ehemaligen DDR hingegen haben sich trotz einer forcierten Industrialisierung aller Räume und trotz standardisierter Bauformen in Stadt und Land die Gegensätze zwischen ländlichen und verstädterten Räumen bis heute eher gehalten.

Nach wie vor zeigen indikatorengestützte Raumanalysen erhebliche Disparitäten zwischen Teilräumen in Deutschland (s. Abb.). Aber das Ergebnis wird nicht vorrangig durch einen Gegensatz „Ländlicher Raum - Verdichtungsraum" geprägt, sondern durch die Unterschiede innerhalb dieser Raumkategorien.

Diese Entwicklungen und Befunde stehen nicht im Einklang mit der mehrheitlichen politischen Wahrnehmung und einer Lobbyarbeit, die sich vorrangig auf Besitzstandswahrung bei Fördermitteln und Transfers richtet. Politisch und institutionell sind in Deutschland die ländlich geprägten Räume überrepräsentiert. Es gibt wesentlich mehr Landräte und Bürgermeister als Oberbürgermeister und die Mehrzahl der Abgeordneten in den Parlamenten versteht sich als Vertreter ländlich geprägter Wahlkreise. Gleichzeitig treten die Lobbyisten ländlicher Räume selbstbewusst und oft fordernd auf. Dies ist ein legitimes Verhalten, doch ist es genauso legitim, nach den sachlichen Begründungen für Förderungen und nach dem volkswirtschaftlichen Ertrag einer Betonung ländlicher Entwicklungserfordernisse zu fragen. 


\section{Strukturunterschiede in und zwischen Raumkategorien}

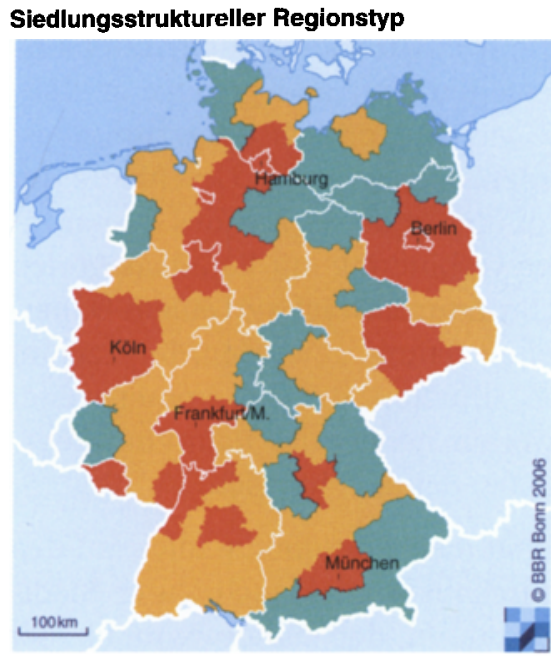

Regionstyp

Agglomerationsrāume

Verstädterte Răume

Lăndliche Răume
Demographische Entwicklung nach Regionstypen

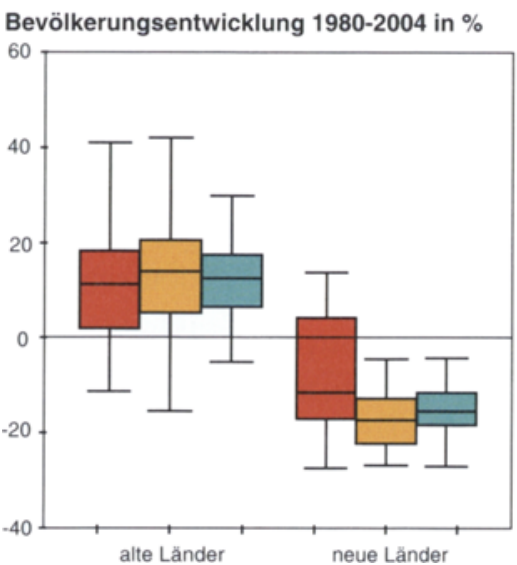

Binnenwanderungssaldo

Durchschnitt 2003/2004 je 1000 Einwohner

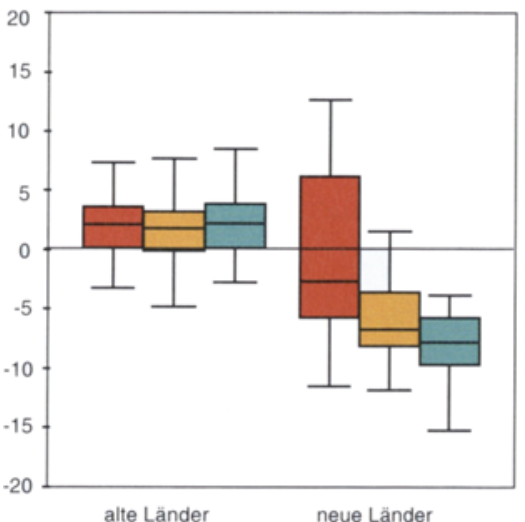

Lebend Geborene 2004 je 1000 Einwohner

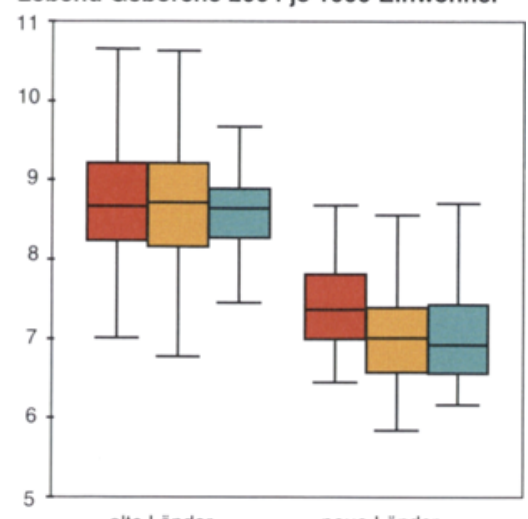

alte Lānder

neue Länder

Lebenserwartung der Männer Durchschnitt 2003/2004 in Jahren

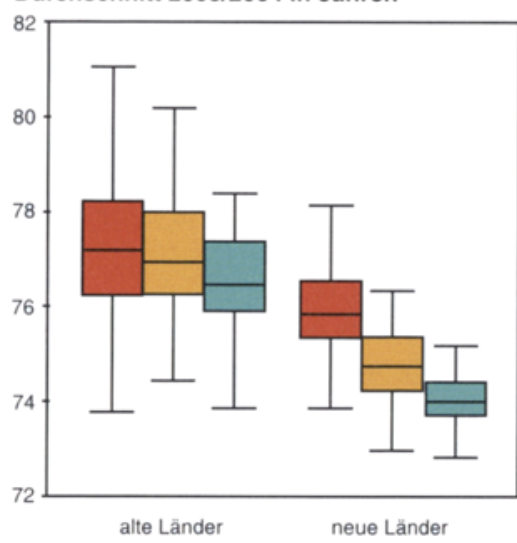

\section{Erläuterung}

Der seit längerem bekannte, aber wenig beachtete empirische Befund, nach dem die Ausprägungen demographischer und ökonomischer Leitindikatoren innerhalb der gebräuchlichen Raumkategorien (Agglomerationsräume, verstädterte Räume, ländliche Räume; $s$. Karte) breiter streuen als zwischen diesen Kategorien lässt sich anhand so genannter Box-Plots eindrucksvoll darstellen. Dabei wird die Spannbreite zwischen kleinstem und größtem Indikatorwert dargestellt, sowie farblich hervorgehoben der Bereich, in dem die Hälfte aller Beobachtungen liegt (Box). Innerhalb der Box ist der Mittelwert gekennzeichnet, hier in Form des Medians, also desjenigen Wertes, oberhalb und unterhalb dessen gleich viele Beobachtungen liegen. Räumliche Bezugseinheit der dargestellten Verteilung der Indikatorwerte sind Kreisregionen.

Aufgegliedert nach den drei Raumkategorien sowie nach alten und neuen Ländern zeigt sich, dass es in Westdeutschland kein signifikantes demographisches oder wirtschaftliches Gefälle zwischen Agglomerationsräumen, verstädterten Räumen und ländlichen Räumen gibt. Die Mittelwerte der Bevölkerungsentwicklung, der Geburtenraten und der Wanderungssalden liegen praktisch gleichauf, während der Abstand zwischen Minimum und Maximum innerhalb aller drei Raumkategorien ein Vielfaches der Abstände zwischen den Mittelwerten ausmacht. Bei der Lebenserwartung gibt es noch ein gewisses Stadt-Land-Gefälle (Städter leben im Durchschnitt etwas länger!), aber auch hier liegen die Extremwerte innerhalb der Agglomerationsräume und der ländlichen Räurne wesentlich weiter auseinander als die jeweiligen Mittelwerte.

Ähnliches gilt für wirtschaftliche Indikatoren, bei denen in Westdeutschland bezüglich Wirtschaftswachstum und Arbeitsmarktsituation die ländlichen Räume im Durchschnitt sogar etwas besser dastehen als die Agglomerationen, jedoch gleichzeitig die Unterschiede innerhalb der Raumkategorien dominieren. Beim verfügbaren Einkommen der Haushalte zeigt sich zwar ein (erwartetes) Stadt-LandGefälle, das allerdings durch ein noch größeres Gefälle bei den Lebenshaltungskosten (Indikator: Nettokaltmiete) weitgehend kompensiert werden dürfte.

Im Gegensatz zu den alten Ländern weisen die neuen Länder in beinahe allen angesprochenen Bereichen noch deutliche Stadt-Land-Gegensätze auf, wobei der ländliche Raum jeweils schlechter abschneidet. Es hat hier offensichtlich bisher keine vergleichbare Annäherung der Lebensverhältnisse stattgefunden wie im Westen.

Fazit: Die gängigen Raumkategorien sind in den alten Ländern für vergleichende Raumanalysen und zur Identifikation siedlungsstrukturell bedingter Entwicklungsunterschiede bzw. -probleme nicht mehr brauchbar. In den neuen Ländern ist ein Gefälle zwischen Stadt und Land zwar noch zu beobachten, doch sind auch dort die Abstände zwischen den Durchschnittswerten der Kategorien kleiner als deren innere Streuung. Für problemorientierte Raumanalysen müssen deshalb Agglomerationsräume ebenso wie ländliche Räume weiter untergliedert und differenziert betrachtet werden. 

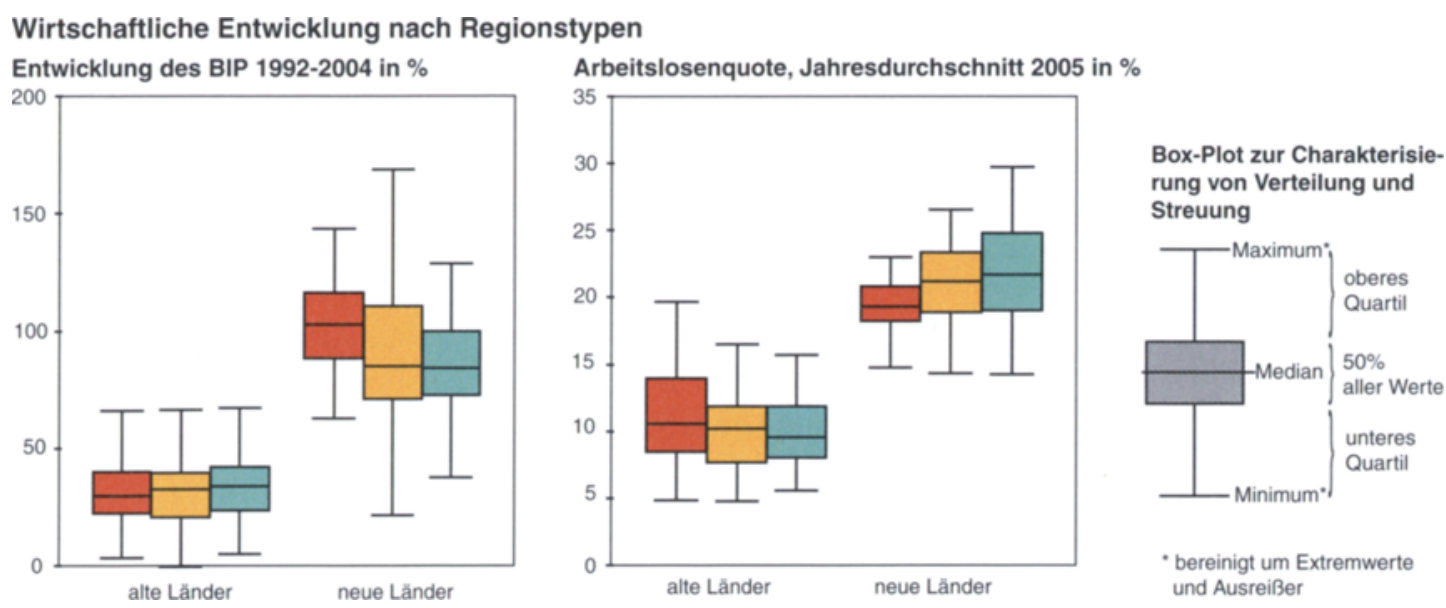

Vertügbares Einkommen der Haushalte im Durchschnitt 2002/03 in $1000 €$ je Haushalt

Durchschnittliche Nettokaltmiete

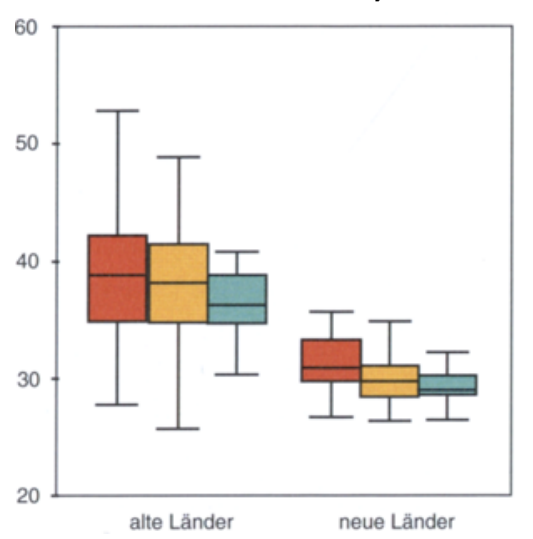

im Durchschnitt 2003/04 in $€$ je $\mathrm{m}^{2}$

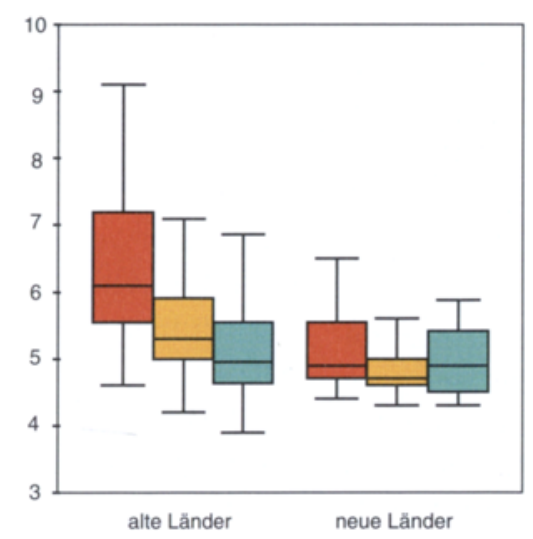

Kreisregionen in

Agglomerationsrăumen

$\square$ Verstädterten Răumen

Lăndlichen Răumen
Quelle:
Laufende Raumbeobachtung
des BBR - Kreisregionen
OBBR Bonn 2006
Die Diskussionen im Leitbildprozess wurden nicht zuletzt auch von den gesellschaftlichen Debatten über die Staatsverschuldung und die Finanzierung des Sozialstaats beeinflusst. So wurde die Formel, „vor der Diskussion über eine Verteilung des Sozialprodukts muss seine Erwirtschaftung stehen“ (sinngemäß beiZimmermann 2003, S. 32 f.), verstärkt in die Raumentwicklungsdiskussion eingebracht, indem gefordert wurde, Raumentwicklungspolitik müsse unter den aktuellen sozioökonomischen Bedingungen das gesamtwirtschaftliche Wachstum unterstützen.

Unter dem Einfluss der Metropolregionendiskussion kam es dann im Leitbildprozess zunächst zu der Idee, eine explizite Metropolregionenpolitik zu entwickeln, denn von den Metropolrergionen wird erwartet, sie seien Motoren von wirtschaftlicher Entwicklung und Innovation (BMBau 1995, S. 27). Das hätte eine explizite Abkehr vom Disparitätenabbau und der Förderung ländlicher Räume bedeutet. Beim zweiten Workshop wurde ein entsprechender Input für neue handlungsorientierte Raumkategorien (Metropolregionen, Brückenräume, ländlich geprägte Räume) eingebracht. Dies erwies sich in den Diskussionen jedoch nicht als tragfähig. So wurde der Gedanke handlungsorientierter Raumkategorien in den Leitbildern zugunsten aufgabenorientierter Leitbilder aufgegeben. Aus „stärke die Starken“ als Ansatz wurde „stärke die Stärken“, in dem Metropolregionen zwar ihren gewichtigen Platz haben, aber nicht alles sind.

Trotz einer klaren Absage an eine schlichte Umpolung der traditionellen Ländlicher-Raum-Politik in eine Metropolregionenpolitik wurden von Kritikern immer wieder entsprechende Vorwürfe geäußert. Schon die Tatsache, dass der ländliche Raum als vertraute Problemkategorie und Handlungsebene der Raumordnung nicht in einem eigenen Leitbild behandelt wird, scheint auszureichen, um von einem Paradigmenwechsel und einer Abkehr vom Gleichwertigkeitspostulat $\mathrm{zu}$ sprechen. Manche Raumordner werten dies als Verrat an den ureigensten Zielen der Raumordnung und manche Vertreter des ländlichen Raums als Abwendung von ausgleichspolitischen Zielen, obwohl die unterschiedlichen Problemstellungen und Entwicklungspotenziale ländlich geprägter, peripherer und dünn besiedelter Räume in allen drei Leitbildern angesprochen und mit Handlungsperspektiven versehen werden. 
Unseres Erachtens wird damit nur auf eine nicht mehr zeitgemäße systematische Bevorzugung einer Raumkategorie verzichtet. Tatsächlich stellt der ländliche Raum - wie bereits dargestellt - schon seit langem keine homogene Problemkategorie mehr dar. Die wirtschaftlichen, sozialen, ökologischen und kulturellen Unterschiede zwischen den Regionen, die aufgrund geringer Bevölkerungsdichte und relativ peripherer Lage zum ländlichen Raum gezählt werden, sind inzwischen so groß, dass eine Gleichbehandlung als raumordnerische bzw. verteilungspolitische Problemkategorie nicht mehr sinnvoll ist. Ähnliches gilt auch für Ballungsräume, die sich gerade in ökonomischer Hinsicht stärker voneinander unterscheiden als vom Durchschnitt der geringer verdichteten Regionen. Von daher erschien es gerechtfertigt, nicht mehr die überkommenen, von der Bevölkerungsdichte bestimmten Raumkategorien zum Ausgangspunkt raumordnungspolitischer Leitbilder zu machen, sondern wirtschafts- und gesellschaftspolitische Problemstellungen, die ihren Niederschlag auf unterschiedliche Weise in allen Raumkategorien finden. Dies gilt zunächst für die räumlichen Ansatzpunkte einer Wachstumsstrategie, im Weiteren aber auch für die anderen Leitbilder.

Gleichwohl hat genau diese neue Sicht- und Darstellungsweise im politischen Raum bisher die heftigsten Reaktionen gegenüber den neuen Leitbildern hervorgerufen. Diejenigen Ressorts und Verbände, die an einer Fortführung der ausgleichenden Strukturpolitik für ländliche Räume besonders interessiert sind, bezichtigten die Raumordnungspolitik einer strategischen Kehrtwende, mit der beabsichtigt sei, Fördermittel massiv von den strukturschwachen ländlichen Räumen in die Metropolregionen umzusteuern. Zweifellos muss bei der Ausgestaltung von Förderinstrumenten auf nationaler ebenso wie auf europäischer Ebene darüber nachgedacht werden, wie neben den strukturschwachen ländlichen Räumen zukünftig auch die Wachstumsmotoren gestärkt werden können. Entsprechende Vorschläge unter dem Stichwort „Lissabon-Strategie“ gibt es inzwischen von der Europäischen Kommission und vom Europäischen Parlament, das sich besonders für die "städtische Dimension“ der zukünftigen Strukturpolitik einsetzt.

Dabei sind die Mittel, die im Rahmen von Investitionsund Förderpolitiken ausgegeben werden können, insgesamt begrenzt, so dass räumliche Prioritätensetzungen unumgänglich sind. Ebenso klar scheint aber, dass unter den Vorzeichen des europäischen Integrationsprozesses und der globalen wirtschaftlichen Verflechtung weder auf eine Entwicklungspolitik zugunsten der Räume mit entsprechenden Potenzialen, wie z.B. der Metropolen und ihrer Verflechtungsräume, noch auf eine Ausgleichspolitik zur Unterstützung und Heran- führung der Peripherie verzichtet werden kann. Beides bedingt sich gegenseitig.

Die Metropolen müssen sich dabei als Zentren und Bezugspunkte regionaler Netzwerke verstehen, die ihre Ziele nur in Kooperation mit den Partnern in ihren Verflechtungsräumen erreichen können. Auf diese Weise sollten auf längere Sicht selbstorganisierte regionale Verantwortungsgemeinschaften entstehen, die die vorhandene administrative Raumgliederung der Länder und Regionen zwar nicht ersetzen, aber sinnvoll ergänzen können.

\section{Fazit: Das Aufgabenverständnis der Raumordnung wird neu akzentuiert}

Mit den sich abzeichnenden neuen Leitbildern wurde wiederholt die Frage nach einem Paradigmenwechsel aufgeworfen. So stellten beispielsweise ARL und ILS NRW die Planertagung 2004 unter die Überschrift „Ausgleich versus Wachstum - Paradigmenwechsel in der räumlichen Planung?“. Dies war noch als offene Frage gemeint. Weiter gingen manche Kritiker, die das Ergebnis des Leitbilddiskurses als einen Paradigmenwechsel einstuften, um damit deutlich zu machen, dass es ihnen inhaltlich zu weit ginge. So kann man festhalten, dass im Laufe des Diskurses der Begriff „Paradigmenwechsel“ durchaus gezielt eingesetzt wurde, um die Ergebnisse zu kritisieren. Ob die Leitbilder wirklich einen Paradigmenwechsel einleiten oder ob es sich nicht doch um Akzentuierungen des etablierten Aufgabenverständnisses handelt, muss die Zeit zeigen.

Wichtiger als diese Bewertung sind die Inhalte der Leitbilder. Aus unserer Sicht ist die Raumordnungs- und Raumentwicklungspolitik gefordert, ihre Aufgabenschwerpunkte und Handlungsansätze an den großen ökonomisch-gesellschaftlichen Fragestellungen auszurichten. Dabei ist Raumordnung sowohl über ihre traditionellen Grundsätze zur Gleichwertigkeit der Lebensverhältnisse als auch durch das jüngere Nachhaltigkeitspostulat einem breiten Spektrum an Herausforderungen verpflichtet, die niemals alle gleichzeitig eingelöst werden können, sondern einer wechselnden zeitlichen und räumlichen Prioritätensetzung bedürfen. Abwägung - ein zentrales Prinzip der Raumordnung - impliziert immer auch solche sachlich begründeten Prioritätensetzungen für bestimmte Zeiträume und Rahmenbedingungen.

Aufbauend auf diesem Verständnis zeichnete sich im Leitbilddiskurs ein weitgehender fachlicher Konsens über die Notwendigkeit einer Neuausrichtung im Aufgabenverständnis der Raumordnung ab, der mit folgenden Schwerpunktsetzungen (vgl. auch MKRO 2006) verbunden ist: 
- Stärkung des Entwicklungsauftrags: Räumliche Entwicklungspolitik wird als eigenständiges Aufgabenfeld betont, das einen Beitrag für Wachstum und Innovation leisten soll. Es zielt darauf ab, „Stärken zu stärken“, statt den Entwicklungsauftrag ausschließlich in den Dienst des Ausgleichsziels zu stellen.

- Neue Gewichtung des Ausgleichsauftrags: Mit der Stärkung des Entwicklungsauftrags geht eine neue Gewichtung des Ausgleichsauftrags einher. Die fast automatisierte Verknüpfung des Postulats der Sicherung der Gleichwertigkeit der Lebensverhältnisse mit dem Ansatzpunkt Disparitätenabbau wird gelockert. Gefordert wird eine zeitbezogene und flexible Ausformung des Gleichwertigkeitspostulats. Dies impliziert die Akzeptanz von „ausgedünnten Räumen“ und eine Konzentration des Zentrale-Orte-Konzepts durch Anpassung von Erreichbarkeits- und Tragfähigkeitskriterien bei gleichzeitiger Konkretisierung von Qualitätsstandards der Daseinsvorsorge.

- Bekräftigung des Ordnungsauftrags: Die neuen Leitbilder stärken die Abwägungskompetenz von Raumordnung zur Koordinierung von Nutzungsansprüchen, Entwicklungspotenzialen und Schutzinteressen. Dabei geht es darum, Flächenschutz zum Ressourcenmanagement und zur Gestaltung landschaftlicher Vielfalt weiterzuentwickeln.

\section{Anmerkung}

(1)

„Erarbeitung von Grundlagen zur Weiterentwicklung raumordnerischer Leitbilder und Handlungsstrategien“, bearbeitet von Prof. Dr. Jürgen Aring, Büro für Angewandte Geographie, Meckenheim

\section{Literatur}

Aring, J.: Modernisierung der Raumordnung. In: Ausgleich versus Wachstum - Paradigmenwechsel in der räumlichen Planung? Hrsg.: ILS NRW. - Dortmund 2004, S. 39-49 (www.ils-shop.nrw. de/cgi-bin/ilsos/070194?id=WXeiTBkc\&mv_pc=49)

Aring, J.: Der Entstehungsprozess der neuen Leitbilder der Raumentwicklung. Inform. z. Raumentwicklung (2006) 11/12

Aring, J.; Sinz, M.: Neue Leitbilder der Raumentwicklung in Deutschland. Modernisierung der Raumordnungspolitik im Diskurs. DISP 165 (2006) 2, S. 43-60

Bundesamt für Bauwesen und Raumordnung (Hrsg.); Stiens, G. (Bearb.): Szenarien zur Raumentwicklung. Raum- und Siedlungsstrukturen 2015/2040. - Bonn 2003. = Forschungen, Bd. 112

Bundesamt für Bauwesen und Raumordnung - BBR: Raumordnungsbericht 2000. - Bonn 2000. = Berichte, Bd. 7

Bundesamt für Bauwesen und Raumordnung - BBR: Raumordnungsbericht 2005. - Bonn 2005. = Berichte, Bd. 21
Bundesministerium für Raumordnung, Bauwesen und Städtebau - BMBau: Raumordnungspolitischer Orientierungsrahmen. - Bonn 1993

Bundesministerium für Raumordnung, Bauwesen und Städtebau - BMBau: Raumordnungspolitischer Handlungsrahmen. - Bonn 1995

Bundesministerium für Verkehr, Bau und Stadtentwicklung - BMVBS: Leitbilder und Handlungsstrategien für die Raumentwicklung in Deutschland. Verabschiedet von der Ministerkonferenz für Raumordnung am 30.06.2006 (www.bbr.bund.de/ cln_006/nn_22518/DE/ForschenBeraten/Raumordnung/ RaumentwicklungDeutschland/LeitbilderKonzepte/Leitbild_ text.html)

Bundesministerium für Verkehr, Bauen und Wohnen - BMVBW: Stellungnahme der Bundesregierung zum Raumordnungsbericht 2005 des Bundesamtes für Bauwesen und Raumordnung (www. bbr.bund.de/index.html?/raumordnung/raumentwicklung/ rob2005.htm)

BMVBW; BBR; BFAG: Leitbilder und Handlungsstrategien für die Raumentwicklung in Deutschland. Diskussionspapier (zur Leitbild-Konferenz am 01.09.2005 in Bonn). Vervielfält. Broschüre

Ganser, K.: Neue Chancen für die Planung. Kontinuität und Wechsel staatlicher Planungs- und Förderungspolitik. Der Architekt (1983) 2, S. 62-64

Kommission für wirtschaftlichen und sozialen Wandel: Gutachten 1977

Ministerkonferenz für Raumordnung - MKRO: Weiterentwicklung raumordnungspolitischer Leitbilder und Handlungsstrategien. Beschluss der 32. Ministerkonferenz für Raumordnung am 28.04.2005 in Berlin

Ministerkonferenz für Raumordnung - MKRO: Leitbilder und Handlungsstrategien für die Raumentwicklung in Deutschland. Beschluss der 33. Ministerkonferenz für Raumordnung am 30. Juni 2006 in Berlin

Schwaetzer, I.: Aufgaben der Raumordnung in Deutschland. In: Raumordnungspolitik in Deutschland. Wissenschaftliche Plenarsitzung 1994. Hrsg.: ARL. - Hannover 1994. = Forschungs- und Sitzungsberichte, Bd. 197, S. 5-11

Sinz, M.: Steuerungsmöglichkeiten der räumlichen Entwicklung. In: Zukunftsforum Raumplanung. Gemeinsamer Kongress 2001 von ARL und BBR. Hrsg.: ARL. - Hannover 2002. = Forschungsund Sitzungsberichte, Bd. 218, S. 57-62

Von der Heide, H.-J.: Gleichwertigkeit der Lebensverhältnisse. In: Raumordnungspolitik in Deutschland. Wissenschaftliche Plenarsitzung 1994. Hrsg.: ARL. - Hannover 1994. = Forschungs- und Sitzungsberichte, Bd. 197, S. 25-30

Zimmermann, H.: Ausgleich versus Wachstum - eine Balance finden. In: Thüringer Innenministerium (Hrsg.): Thüringer Raumordnungskonferenz 5. September 2003. - Erfurt 2003, S. 19-38

Manfred Sinz

Holtzendorffstraße 8

14057 Berlin

E-Mail: Manfred.Sinz@web.de

Prof. Dr. Jürgen Aring

Büro für Angewandte Geographie

Klosterstraße 84

53340 Meckenheim

E-Mail: aring@t-online.de 